\title{
Gestion des ressources humaines et performance économique : une étude du bilan social
}

Christel DECOCK GOOD ${ }^{1}$ et Laurent GEORGES ${ }^{2}$

\section{Résumé}

Cette recherche examine l'impact de pratiques de gestion des ressources humaines (le climat social, les politiques de rémunération et de formation et les conditions de travail, la structure de l'emploi) sur la performance de l'entreprise. Les résultats sont fondés un échantillon national de 58 entreprises. Le modèle structurel démontre un pouvoir prédictif des dépenses de formation et des conditions de travail.

\section{Mots clés}

Performance sociale - bilan social - comptabilité sociale

\begin{abstract}
This study evaluated the links between systems of work practices and firm performance. We examine the impact of employment structure, social climate, remuneration policy, training expenses and work conditions on financial performance. The results are based on a national sampling of 58 firms. The structural model demonstrates predictive power of the training expenses and work conditions.
\end{abstract}

\section{Keywords}

Social performance - Social accounting

\footnotetext{
${ }^{1}$ Professeur, EDHEC Département Comptabilité Contrôle de gestion, 393 promenade des Anglais BP 3116 06202 NICE Cedex 3. email : christel.decock@edhec.edu

2 Professeur, EDHEC Département Marketing-Vente, 393 promenade des Anglais BP 311606202 NICE Cedex 3. email : laurent.georges@edhec.edu
} 


\section{Introduction}

Quelles relations existent-ils entre la performance sociale et la performance économique des entreprises? Au vu des aspects idéologiques suggérés par cette relation, de nombreux auteurs ont essayé de la tester empiriquement. Si ce débat peut sembler lointain (on se souvient de la provocation de Milton Friedman en $1970^{3}$ ), il ne l'est pas auprès de la communauté académique ou des praticiens. La médiatisation d'événements tels que le sommet sur le développement durable de Johannesburg et la première année d'application de la loi des Nouvelles Réglementations Economiques en 2003, sur la diffusion d'informations sociales et environnementales, ne font que renforcer l'intérêt pour ce type de recherche. Pour autant, alors que ces thèmes sont aujourd'hui au cœur de nombreuses réflexions, les problématiques restent entières. En effet, peu de modèles sont proposés et les études empiriques manquent souvent de précisions. Plusieurs explications peuvent être avancées. Tout d'abord, les difficultés à obtenir des données pertinentes sur des domaines sensibles comme l'écologie et les ressources humaines (RH) confortent ces situations. Ensuite, les relations de causalités étant parfois délicates à établir, les travaux finissent par se cantonner à des états descriptifs. Ainsi, force est de constater que très peu de recherches françaises abordent ce type de problématique : la comptabilité sociale n'a pas connu de fort retentissement, au détriment de la comptabilité environnementale. Par ailleurs, pour les rares travaux existants, les analyses adoptées sont très différentes: un premier groupe de recherches s'est concentré sur la politique sociale de l'entreprise dans son ensemble alors qu'un second traite de la politique salariale et qu'un troisième aborde la performance des ressources humaines à travers un tableau de bord. Au final, bien que ces apports soient très riches il apparaît difficile de les comparer.

Cette rareté des recherches existantes et leur faible complémentarité est particulièrement dommageable. En effet, combler ces déficits, ne serait-ce que partiellement, permettrait au plan théorique d'améliorer notre compréhension des liens unissant la performance sociale et la performance économique. Sur un plan plus managérial, cela éviterait le recours à des règles et heuristiques - plus de formation, moins de pressions sur les salariés - ambiguës et simplificatrices. Ces enjeux théoriques et pratiques mettent en évidence l'importance de la question de la méthodologie de recherche la plus appropriée.

Cet article s'organise donc de la manière suivante. Dans la première partie, nous nous attachons à définir le cadre théorique de notre recherche. Pour y parvenir, nous nous appuyons sur les apports de l'approche Resource Based ainsi que sur les études réalisées dans le domaine de la performance sociale. Après avoir discuté du champ conceptuel, nous sélectionnons différentes variables de performance sociale et économique que nous intégrons au sein d'un modèle explicatif. Pour effectuer ces choix, nous utiliserons notamment un outil de mesure peu exploité à savoir le bilan social. Véritable tableau de bord des ressources humaines, ce document officiel sera au cœur de notre analyse. Dans une troisième partie, nous précisons la méthodologie de notre enquête réalisée sur un échantillon d'entreprises françaises. Cette partie sera suivie d'une présentation détaillée et approfondie des résultats du test de nos hypothèses par la méthode des équations structurelles (analyse des moindres carrés

\footnotetext{
${ }^{3}$ « la responsabilité sociale d'une entreprise est de faire du profit ».
} 
partiels de type PLS). La conclusion soulignera les limites de cette étude, les voies de recherche prometteuses, ainsi que les diverses implications théoriques et managériales.

\section{Le cadre théorique}

Après avoir présenté les apports de l'approche Resources Based, nous nous intéresserons aux contributions issues des travaux sur la problématique de la relation performance socialeperformance économique.

\subsection{L'approche Resources Based}

$\mathrm{Au}$ cours de ces dernières années, la réflexion stratégique s'est orientée vers de nouvelles voies afin de mettre en exergue les origines des écarts de performance pour des entreprises appartenant à un même environnement. La problématique tient au fait que des entreprises d'un même secteur diffèrent les unes des autres et que ces différences perdurent dans le temps. Dans le cadre de l'approche Resources Based, cela signifie que les ressources utilisées ne sont pas les mêmes.

Le terme ressources doit être compris dans un sens large : il peut s'agir d'éléments matériels comme immatériels. Barney (1991) les définit comme «les actifs, capacités, processus organisationnels, informations, connaissances, etc., contrôlés par l'entreprise et qui lui permettent de concevoir et mettre en œuvre des stratégies ». L'idée que la performance individuelle des salariés peut avoir un impact sur les résultats économiques et financiers est un fait acquis chez les praticiens et les chercheurs. L'intérêt dans ce domaine s'est cependant intensifié avec le courant de la théorie de la ressource qui stipule que les employés d'une entreprise constituent un avantage compétitif difficile à dupliquer pour les concurrents. Wright et McMahan (1992) développent quatre critères qui doivent être réunis pour qu'un avantage compétitif émerge des ressources humaines. Premièrement, ces dernières doivent créer de la valeur dans le processus de production de la firme. Deuxièmement, elles doivent constituer une ressource rare. Le troisième critère est que la combinaison des investissements en capital humain au sein de la firme ne soit pas facilement imitable. Enfin, les ressources humaines ne doivent pas être sujet à un remplacement facile par une avancée technologique.

Les pratiques de gestion des ressources humaines (GRH) influencent les compétences des employés à travers l'acquisition et le développement du capital humain de l'entreprise. En proposant des expériences formelles et informelles d'apprentissage, que ce soit des formations de base ou de management, la firme peut influencer le développement de ses employés. Les exemples d'efforts pour motiver ou influencer les comportements comprennent les primes sur performance, individuelles ou collectives, l'utilisation de systèmes de promotion interne fondés sur le mérite, ou d'autres formes incitatives liées aux résultats boursiers.

Par ailleurs, Bailey (1993) stipule que les RH sont fréquemment «sous utilisées » parce que les personnes ne travaillent pas au maximum de leurs capacités. La stratégie organisationnelle pour provoquer un effort supplémentaire de la part des employés est alors susceptible de générer des gains supérieurs aux coûts engendrés. De ce fait, les pratiques salariales peuvent contribuer à cet effort en influençant la motivation, les compétences des employés et la structure qui peut leur fournir la capacité de contrôle de leurs rôles. Cependant, la contribution organisationnelle à la motivation et à la performance est limitée si les emplois sont structurés, 
de telle sorte que les personnes sont présumées connaître leur emploi mieux que quiconque et qu'elles n'ont pas les capacités et les compétences pour évoluer dans de nouvelles voies.

L'approche des resources based suggère clairement que l'attitude d'un employé peut avoir des implications importantes sur la performance globale de l'entreprise. Et les pratiques de gestion des ressources humaines peuvent affecter la performance individuelle des salariés à travers les influences sur ses compétences, sa motivation et les structures organisationnelles mises en place pour améliorer les conditions de l'emploi. Si on en croît cette approche, on peut s'attendre, dans un premier temps, à ce que les pratiques de gestion de ressources humaines incitatives (soit globalement au niveau de l'entreprise, soir au niveau individuel) vont conduire directement à l'amélioration de résultats comme la productivité, le chiffre d'affaires, ou d'autres variables sur lesquelles les employés peuvent exercer un contrôle direct. Dans un second temps, si les retours sur investissement sont plus élevés que les coûts dans les entreprises ayant mis en place de telles pratiques, on peut s'attendre à ce que le turnover des employés soit plus faible et la productivité meilleure ce qui influencera également les indicateurs de performance financière. Ainsi, dans l'anticipation de la présentation de notre modèle nous proposons d'examiner les travaux empiriques antérieurs sur la relation GRH / performance économique fondé sur les deux disciplines que sont les domaines des ressources humaines et de la comptabilité (voir tableau 1).

Tableau 1 Les recherches en GRH et comptabilité

\begin{tabular}{|c|c|}
\hline GRH & COMPTABILITE \\
\hline $\begin{array}{l}\text { Problématique : } \\
\text { préparation et activation optimales des ressources } \\
\text { humaines de l'entreprise }\end{array}$ & $\begin{array}{l}\text { Problématique : } \\
\text { fournir un système d'informations afin de mesurer } \\
\text { l'efficience d'une entité }\end{array}$ \\
\hline $\begin{array}{l}\text { Approches théoriques: } \\
\text { analyses stratégique et psychosociologique, } \\
\text { constructivisme }\end{array}$ & $\begin{array}{l}\text { Approches théoriques : } \\
\text { individualisme méthodologique et rationalité } \\
\text { économique ; constructivisme }\end{array}$ \\
\hline $\begin{array}{l}\text { Perception de l'entreprise : } \\
\text { ensemble coordonné d'individus et de groupes, en } \\
\text { situation de travail animés par des objectifs individuels } \\
\text { et collectifs }\end{array}$ & $\begin{array}{l}\text { Perception de l'entreprise : } \\
\text { ensemble coordonné de moyens techniques et humains, } \\
\text { susceptibles de générer de la valeur }\end{array}$ \\
\hline $\begin{array}{l}\text { Acteurs clés : } \\
\text { individus, groupes et technostructure }\end{array}$ & $\begin{array}{l}\text { Acteurs clés: } \\
\text { parties prenantes internes et externes à l'entreprise }\end{array}$ \\
\hline $\begin{array}{l}\text { Mission des dirigeants : } \\
\text { connaître la réalité et le fonctionnement des équilibres } \\
\text { sociaux de l'organisation et veiller à l'ajustement } \\
\text { permanent entre les objectifs économiques et les } \\
\text { ressources humaines }\end{array}$ & $\begin{array}{l}\text { Mission des dirigeants : } \\
\text { maximiser la création de valeur }\end{array}$ \\
\hline $\begin{array}{l}\text { Objet principal de la discipline : } \\
\text { le salarié, membre de groupes développant sous } \\
\text { contraintes des stratégies construites et évolutives. }\end{array}$ & $\begin{array}{l}\text { Objet principal de la discipline: } \\
\text { Les parties prenantes soucieuses de l'impact de } \\
\text { l'entreprise sur leurs richesses et le dirigeant, son } \\
\text { mandataire, chargé de défendre ses intérêts. }\end{array}$ \\
\hline
\end{tabular}

\subsection{La problématique de la relation GRH - performance économique :}

La problématique de la performance sociale peut être abordée selon plusieurs aspects, selon le champ social étudié. Cependant, les travaux empiriques réalisés dans ce champ sont 
particulièrement rares. En effet, deux difficultés sont à contourner : appréhender la GRH et la rapprocher d'un indicateur de performance économique. Les premières études examinées sont celles liées à la politique sociale de l'entreprise.

\subsubsection{Les études fondées sur la politique sociale}

Les études corrélationnelles entre la politique sociale et performance économique montrent, de façon générale, une association positive. Cependant, les comparer reste délicat car chacune d'entre elles utilise des indicateurs sociaux différents.

Denison (1984) a réalisé une recherche sur 34 entreprises de plusieurs secteurs industriels. Les données sociales proviennent de questionnaires (traités à l'université de Chicago) et relèvent principalement de la prise de décision, l'organisation du travail et le degré d'implication. Les variables économiques sont des variables comptables : rentabilité des actifs (résultat net/actif) et rentabilité commerciale (résultat net sur chiffre d'affaires). Il est intéressant de noter que l'auteur procède à une analyse dynamique en étudiant la corrélation entre les données sociales de la date $t$ et les données financières de $\mathrm{t}$ à $\mathrm{t}+5$.

Schuster (1988) a mené une étude sur 1300 entreprises. L'auteur a pour objectif de tester la relation entre la performance économique mesurée par le rendement des fonds propres et une gestion des ressources humaines «active », à savoir, ayant une politique de rémunération individualisée, une participation aux résultats, une personnalisation des objectifs, une flexibilité des horaires de travail et une pratique de la consultation interne. La relation établie est faible mais positive.

Notons les travaux de Grinyer, Mayes et McKiernan (1990) qui procèdent à une recherche sur 25 entreprises ayant connu un redressement. Des entretiens ont été menés dans ces entreprises à plusieurs niveaux hiérarchiques, ainsi que dans des entreprises n'ayant pas connu de redressement. Il apparaît que dans les entreprises redressées, le personnel, la communication interne et les relations industrielles sont les trois domaines les plus investis par le management.

Enfin, Huselid (1995) met en évidence une relation entre une gestion des ressources humaines de qualité (ayant reçu le label américain de High Performance Work Practices) et la performance financière. L'auteur a construit une échelle de mesure de cette gestion de qualité, relative aux items suivants : sélection du personnel, valorisation de la performance, moyens incitatifs, service de recherche, procédures de doléances, partages de l'information, valorisation de l'attitude, la participation des ressources humaines au management, l'intensité des efforts de recrutement, le nombre moyen d'heures de formation par employé par an et les critères de promotion interne (ancienneté contre mérite). L'étude porte sur un échantillon de 968 entreprises. Les indicateurs économiques sont des données comptables et boursières. Les résultats obtenus sur la relation entre performance sociale et économique restent cependant ambigus.

\subsubsection{Les études fondées sur la politique salariale}

La deuxième catégorie d'études est relative aux politiques sociales.

Les politiques salariales diffèrent d'une entreprise à l'autre en fonction de leurs priorités. On peut donc être amené à constater des disparités sur des postes présentant des compétences plus ou moins recherchées par les organisations. Ces différences ne sont relatives qu'à certaines particularités de qualifications demandées et ne s'appliquent pas à l'ensemble des employés. Elles sont par ailleurs davantage liées aux caractéristiques d'une entreprise plutôt qu'à celles d'un secteur d'activité. 
Les travaux qui ont pour objectif de présenter une relation entre la politique salariale et la performance de l'entreprise peuvent être analysés selon le prisme suivant :

- il faut distinguer les cadres supérieurs et des autres salariés ;

- il est à considérer que le niveau du salaire n'est pas la seule dimension à retenir : sa composition est toute aussi importante, si ce n'est plus.

Les politiques de rémunérations des cadres supérieurs sont des thèmes très présents autant auprès des académiciens en comptabilité comme en GRH, qu'auprès des praticiens à travers celui des stocks options. Cette problématique est pourtant ancienne : McGuire et al (1962), Lewellen et Huntsman (1970), Masson (1971), Magnan et al (1993) ont procédé aux premières études empiriques dans ce domaine. Ces études montrent une forte corrélation entre le salaire des cadres et la taille des entreprises mais moins nettement avec des ratios ou données comptables. Les travaux plus récents (Wilkinson et al, 1994 ; Botosan \& Plumlee, 2001 ; Bens et al, 2002) identifient davantage le coût des stocks options et remettent en cause son rôle premier : la motivation des cadres dirigeants.

\subsubsection{Les évaluations de la performance sociale : la comptabilité des ressources humaines}

La troisième catégorie de recherche porte davantage sur les évaluations de cette performance. Les principaux travaux sur la comptabilité sociale ou encore la comptabilité des ressources humaines proviennent de Flamholtz $(1971,1988)$. L'auteur fonde son raisonnement sur deux dimensions :

- la valeur conditionnelle attendue d'un individu dans une organisation E(CV);

- la valeur réalisable attendue d'un individu dans une organisation $E(R V)$.

$\mathrm{E}(\mathrm{CV})$ est la valeur potentielle maximale attendue qui peut provenir d'un individu pour l'organisation, sous l'hypothèse qu'il ne quitte pas cette organisation. Elle représente donc la valeur de ses services. E(RV) est la valeur attendue d'un individu dans l'organisation, sachant qu'il existe une probabilité non nulle pour que la personne quitte l'organisation. La différence entre ces deux valeurs correspond alors au coût du turnover. Ces espérances mathématiques peuvent ensuite être traduites en unités monétaires en se référant à la valeur du service rendu par l'individu, qui peut être le coût de ce service, sa valeur de marché ou la part de bénéfice provenant de l'individu lié à son travail. Cette difficile valorisation fait que la méthode a été jusqu'à ce jour peu utilisée.

Comme le souligne Stabile (1993), la comptabilité sociale ${ }^{4}$ évolue peu car la profession comptable n'est pas soumise à des pressions pour la faire évoluer dans ce sens. Que ce soit dans les pays anglo-saxons (où ce sont des organismes privés qui établissent les réglementations comptables en vue de satisfaire les attentes des investisseurs), ou bien dans les pays continentaux (où le pluralisme des utilisateurs est davantage reconnu), aucun groupe de pression n'a permis à la comptabilité sociale de se développer.

Plus généralement, selon Roslender (2000), la comptabilité sociale souffre de l'ambiguité entre les locutions : capital humain et actif humain. L'auteur propose la différenciation de différents termes mais nous semble échouer par son manque de précision. Par actif, on entend l'ensemble des capacités intangibles des ressources humaines constituant l'entreprise alors que le capital humain pourrait se définir comme un actif intellectuel, en sus des actifs tangibles et intangibles. Nous préférons l'approche de D'Arcimoles et Fakhfakh (1997, p 65), pour qui le capital humain peut se définir comme « la connaissance précise des procédures ou

\footnotetext{
${ }^{4}$ voir définition ci-après.
} 
la maîtrise de savoir-faire locaux particulièrement précieux pour l'entreprise. Cette relation justifie des efforts engagés par les entreprises pour construire ce capital, dont les coûts et les avantages sont partagés entre employeur et employé ». Cependant, ces concepts restent difficilement mesurables.

Les difficultés posées par cette comptabilité sociale invitent à penser que cette dernière n'est pas en mesure de renseigner les investisseurs ou analystes extérieurs dans leur appréciation d'un risque social éventuel. La valorisation monétaire de telles ressources peut sembler très subjective et étriquée dans la mesure où l'ensemble des risques ne sont pas pris en compte (d'Arcimoles, p130).

De ce fait, un tableau de bord social semble davantage adapté. La même perception est soutenue par Capron (1995), pour qui la comptabilité sociale a trois fonctions majeures :

- fournir une information chiffrée concernant les individus en tant que membres d'une organisation ;

- servir de cadre d'aide à la décision dans les politiques de gestion des ressources humaines ;

- motiver les décideurs et influencer leur perception des ressources humaines.

Ainsi, en accord avec Martory (1999), la comptabilité soci(ét)ale n'est pas tant l'inscription au bilan et au compte de résultat des ressources humaines mais la mise en lumière de valeurs immatérielles. On rejoint alors la définition de Mathews (1997, p.483) : «la comptabilité sociale et environnementale est définie comme les diffusions volontaires d'informations, à la fois qualitative et quantitative faites par les organisations pour informer ou influencer les audiences. Les diffusions quantitatives peuvent être en des termes financiers ou non financiers $»^{5}$.

\section{Choix des variables étudiées, modèle théorique et hypothèses de recherche}

Après avoir sélectionné les différentes variables nous permettant d'appréhender les notions de performance économique et de performance sociale, nous présentons notre modèle théorique ainsi que nos hypothèses de recherche.

\subsection{Le choix des variables à étudier}

\subsubsection{Le bilan social et le choix des variables sociales}

En France, nous disposons d'un précieux outil d'analyse : le bilan social. Celui-ci trouve son origine dans la loi du 12 juillet 1977 qui oblige toute entreprise de plus de 300 salariés de l'établir et de le soumettre au comité d'entreprise. Ses objectifs sont d'améliorer le système d'informations sociales envers les différents acteurs (salariés et syndicats), de planifier la gestion des ressources humaines, de favoriser le dialogue et de comparer dans le temps les

\footnotetext{
${ }^{5}$ Notre traduction de «social and environmental accounting has been defined as...voluntary disclosures of information, both qualitative and quantitative made by organizations to inform or influence a range of audiences. The quantitative disclosures may be in financial or non financial terms ».
} 
données. Ce document a été conçu dans une approche macro-économique afin d'apprécier les mesures prises en faveur de l'emploi (Comhaire \& Dendauw, 1998) et de servir d'instruments de repérage et d'alerte pour les observateurs externes ainsi que d'éléments de réflexion pour les acteurs internes.

Ce document est établi selon la logique suivante (Iacono, 1996).

1. La première idée-force du bilan social est relative à son découpage temporel, calqué sur la démarche comptable et financière : il est établi annuellement.

2. La deuxième idée-force est la démarche méthodologique sous-jacente à ce document. Il s'inscrit dans une démarche entièrement analytique. Le «bilan» ne retranscrit pas un solde positif ou négatif relatif à la politique sociale de l'entreprise mais présente plutôt un état des lieux de l'organisation, une représentation de l'existant.

3. La troisième idée-force est la logique financière de ce bilan. En effet, après une simple lecture, il est facile d'identifier les coûts des ressources humaines et de connaître la masse salariale de l'entreprise. Cependant, il est très délicat de porter un jugement sur la politique sociale menée et sur les impacts de cette politique sur l'enrichissement et la création de valeur de l'organisation. Le bilan social souffre de l'absence d'évaluation potentielle d'une performance. Les indicateurs ne rendent pas compte du bien fondé des actions menées en terme d'embauches, de maintien d'emploi, de formation ou de conditions de travail. Seules les comparaisons temporelles ou relatives à d'autres entreprises fournissent des éléments de réponse.

Plus précisément, ce bilan est composé de 7 chapitres et 34 rubriques qui sont les suivantes :

- l'emploi (effectifs, travailleurs extérieurs, embauches, départs, chômage et absentéisme) ;

- les rémunérations et les charges accessoires (la masse salariale, la hiérarchie des rémunérations, les charges accessoires, la charge salariale et la participation financière) ;

- les conditions d'hygiènes et de sécurités (les accidents du travail et de trajet, les maladies professionnelles et les dépenses de sécurités);

- les autres conditions de travail : la durée du travail, son aménagement horaire, son organisation et les dépenses d'amélioration de ses conditions ;

- la formation: le pourcentage de la masse salariale consacré à la formation continue, le nombre de stagiaires, le nombre d'heures de stage, les congés formation ;

- les relations professionnelles : la composition du comité central d'entreprise, le nombre de ses réunions, les dates et les objets des accords ;

- les autres conditions de vie relevant de l'entreprise : les œuvres sociales et le coût des autres prestations complémentaires

Ces rubriques ont pour objectif de privilégier les chiffres et l'objectivité que ces derniers suggèrent. Le résultat en est que le document est malheureusement difficilement lisible ! L'abondance de chiffres n'est pas compensée par des commentaires, ce qui laisse une impression de collecte de données sans analyse. Les travaux de Lequin (1989) donnent 
davantage de visibilité sur ces indicateurs puisque l'auteur propose une analyse factorielle qui permettrait de passer de 74 à 47 indicateurs.

Par ailleurs, les données sont présentées en dehors de tout contexte stratégique et économique : les contraintes extérieures sont complètement ignorées. De ce fait, il est difficile pour tout investisseur extérieur à l'entreprise de porter un jugement sur la politique salariale menée. Il en est de même pour le climat social. En outre, académiciens et praticiens sont en accord pour souligner l'importance du climat social au sein d'une entreprise et de voir en cette dimension un déterminant important de l'équilibre interne. L'absence de commentaires est, pour ce point, très dommageable.

\subsubsection{Les indicateurs traditionnels de performance économique et financière}

Les variables utilisées dans les recherches antérieures sont de deux ordres : les indicateurs comptables et les indicateurs issus des marchés financiers. Ces derniers sont appropriés lorsque l'étude porte sur la réaction des investisseurs à une information nouvelle, dans le cadre de la théorie financière néo-classique. N'ayant pas adopté cette perspective, nous nous sommes concentrés sur les indicateurs comptables.

Dans l'état de l'art, les principaux indicateurs recensés sont les suivants :

- le ratio résultat sur capitaux propres (Abbott et Monsen, 1979 ; Bowman et Haire, 1975 ; Freedman et Jaggi, 1992 ; Davidson III et Worrell, 1990) ;

- le ratio résultat sur total des actifs (Freedman et Jaggi, 1992 ; Hackston et Milne, 1996 ; Davidson III et Worrell, 1990 ; McGuire, Sundgren et Schneeweis, 1988) ;

- le taux de marge d'exploitation (Freedman et Jaggi, 1992 );

- le ratio cash-flow sur total des actifs (Freedman et Jaggi, 1992);

- le ratio cash-flow sur capitaux propres (Freedman et Jaggi, 1992 ) ;

- la capitalisation boursière (Hackston et Milne, 1996) ;

- le taux de croissance du résultat par action (Sturdivant et Ginter, 1977) ;

- le taux de croissance du cours de l'action sur 2 et 5 ans (Alexander et Buchholz, 1978) ;

- le taux de croissance du ratio résultat sur capitaux propres (Roberts, 1992).

Au regard de ces études, il s'avère que de meilleurs résultats ont été obtenus avec des données comptables et financières, plutôt que des indicateurs boursiers : trop de paramètres peuvent intervenir dans la variation du cours de l'action d'une entreprise et les pratiques de gestion des ressources humaines pour prétendre une corrélation entre ces deux grandeurs. Cependant, le choix d'une variable comptable reste délicate. En effet, les actionnaires veulent connaître la valeur de leur entreprise et la capacité des dirigeants à contribuer à cette augmentation de valeur. En revanche, les préteurs et créanciers vont davantage chercher à estimer la capacité de l'entreprise à générer des flux de trésorerie.

De ce fait, les indicateurs comptables ont plusieurs vocations : renseigner sur la valeur de l'entreprise et sa capacité de croissance mais aussi renseigner sur les flux de trésorerie futurs. De ce point de vue, Cormier et al (2001) examinent la pertinence de plusieurs indicateurs de performance : le résultat net, les flux de trésorerie d'exploitation; le résultat résiduel et la valeur ajoutée.

Le résultat net est une grandeur assez contestée puisqu'il n'est pas exempt de choix comptables pouvant dépendre des stratégies mises en place par les dirigeants. Par ailleurs, il s'avère contesté comme représentation de la performance globale des entreprises. Le résultat d'exploitation, notamment utilisé par Freedman et Jaggi (1992), est davantage pertinent car il traduit la capacité de l'entreprise à générer un profit par son activité, 
Les flux de trésorerie constituent un indicateur de mesure de performance alternatif. Bien que leur contenu informationnel soit faible (Bowen et al, 1986), il s'agit d'indicateurs complémentaires à ceux plus traditionnels comme le résultat net (Janin, 2002). Ces résultats sont dans le sens choisi par le FASB, organisme de normalisation comptable américain. En effet, aux Etats Unis, la présentation d'un tableau de flux de trésorerie est obligatoire. La norme internationale IAS 7 recommande également une présentation de ce tableau. Et en France, depuis 1999, le tableau des emplois ressources peut être désormais présenté sur la notion de trésorerie (à la place de celle de fonds de roulement).

La notion de valeur ajoutée, très présente dans la comptabilité française, repose sur la notion de distribution de richesse aux différents partenaires de l'entreprise. Pour reprendre l'expression de Cormier et al (2001, p.84) : «la valeur ajoutée est une façon simple et immédiate de voir le bénéfice comme le résultat des efforts collectifs du capital, de la gestion, de l'Etat et des salariés ».

Enfin, la dernière grandeur examinée pour mesurer la performance est le résultat résiduel, à savoir le résultat net moins le coût du capital investi. Ce résultat résiduel, développé notamment par Bernard (1995) proche, dans sa construction de l'EVA. Bien que cet indicateur ne soit pas exempt d'un certain nombre de limites (Biddle et al, 1998), il permet d'approcher un autre aspect de la performance.

Cette revue de la littérature permet de dégager les deux points suivants. Tout d'abord, l'évaluation des ressources humaines passe davantage à travers un tableau de bord plutôt qu'une évaluation en terme de coûts. Ensuite, le choix de la variable de performance économique est tout aussi déterminante que celles de la GRH.

\subsubsection{Les données et les variables retenues}

Les données sociales proviennent des résultats de demandes par courrier auprès des grandes entreprises françaises. Elles ont été intégrées à une d'une base de données (collecte de bilans sociaux) constituée par nos soins et utilisées pour les travaux statistiques. La quantité d'informations disponible dans le bilan social a nécessité d'effectuer un choix. Les variables retenues ont été sélectionnées selon les trois critères suivants :

- données simples et peu discutables ;

- données reflétant au mieux les éléments mis en évidence dans les recherches empiriques antérieures, à savoir, les niveaux de rémunération, les conditions de travail, la structure des effectifs et le comportement du personnel ;

- données les plus fréquemment disponibles.

Finalement, 16 variables sociales ont été retenues pour appréhender 5 concepts (cf. Tableau 2) :

- $\quad$ structure et emploi (4 variables);

- rémunérations (3 variables);

- formation (3 variables) ;

- conditions de travail (2 variables) ;

- climat social (5 variables). 
Les données économiques, permettant d'appréhender le concept performance, sont issues des rapports annuels et de bases de données (Diane). Leur choix s'avère en revanche plus délicat. Nos critères de sélection pour le choix de ces données sont de deux ordres :

- le caractère économique de la variable ;

- l'originalité de la variable par rapport aux recherches antérieures.

Compte tenu des travaux récents, nous avons retenu trois variables pour mesurer la performance :

- les flux de trésorerie d'exploitation ;

- la valeur ajoutée ;

- le résultat résiduel.

Tableau 2 : Concepts et variables retenus

\begin{tabular}{|c|c|c|c|}
\hline Concept & Variables & Sigles & Calculs \\
\hline \multirow[t]{3}{*}{ Rémunérations } & \begin{tabular}{|l|} 
Ecart de rémunération \\
moyenne ouvrier/cadres
\end{tabular} & ERMO & $\begin{array}{l}\text { Rémunérations moyennes des } \\
\text { cadres/Rémunérations moyennes ouvriers }\end{array}$ \\
\hline & \begin{tabular}{|l|} 
Rémunération moyenne \\
cadres
\end{tabular} & RMC & Rémunérations moyennes cadres \\
\hline & 10 salaires supérieurs & RMSUP & Moyenne des 10 salaires supérieurs \\
\hline \multirow[t]{3}{*}{ Formation } & $\%$ dépenses de formation & FORM & Dépenses de formation/total salaires \\
\hline & $\%$ stagiaires cadres & STAG & Total stagiaires/total effectif \\
\hline & $\begin{array}{l}\text { Heures de stages payées } \\
\text { par personne }\end{array}$ & HSP & Total heures de stages/effectif total \\
\hline \multirow[t]{3}{*}{ Conditions de travail } & \begin{tabular}{|l|} 
Taux de gravité des \\
accidents du travail
\end{tabular} & TGA & $\begin{array}{l}\text { Nombre de jours perdus en accidents du } \\
\text { travail/(effectif total*200) }\end{array}$ \\
\hline & Euvres sociales & OS & Dépenses sociales/effectif total \\
\hline & Durée du travail & HHM & Horaire hebdomadaire moyen \\
\hline \multirow[t]{5}{*}{ Climat social } & Taux d'absentéisme & TXA & \begin{tabular}{|lccc} 
Nombre & de & jours & perdus \\
absentéisme/(effectif total $* 200)$ & \\
\end{tabular} \\
\hline & Taux de démission total & TDT & Total démission /effectif permanent \\
\hline & \begin{tabular}{|l|l|}
$\begin{array}{l}\text { Taux de démission des } \\
\text { cadres }\end{array}$ & \\
\end{tabular} & TDC & $\begin{array}{l}\text { Démission des cadres /Effectif permanent } \\
\text { des cadres }\end{array}$ \\
\hline & $\begin{array}{l}\text { Taux de démission des non } \\
\text { cadres }\end{array}$ & TDNC & $\begin{array}{l}\text { Démission des non cadres/Effectif } \\
\text { permanent non cadre }\end{array}$ \\
\hline & \begin{tabular}{|l|}
$\begin{array}{l}\text { Ecart de rémunération } \\
\text { hommes femmes cadres }\end{array}$ \\
\end{tabular} & ERHF & $\begin{array}{l}\text { Rémunération des } \\
\text { cadres/rémunération des hommes cadres }\end{array}$ \\
\hline \multirow[t]{3}{*}{ Structure et emploi } & $\%$ cadres & PGCA & Effectifs cadres/Effectif total \\
\hline & Taux d'embauche & TXE & \begin{tabular}{|l} 
Nombre d'embauches \\
permanent
\end{tabular} \\
\hline & Taux licenciement & TXL & Nombre de licenciements/effectif permanent \\
\hline
\end{tabular}

Nous pouvons regretter que ces données ne donnent que des informations incomplètes quant à la composition des rémunérations. En effet, nous ignorons les parts fixes et variables ainsi que les primes éventuelles et leurs critères. Nous n'avons également que trop peu de renseignements sur les promotions internes pour pouvoir retenir ce critère, qui pourtant, aurait été intéressant. 
Il en est de même pour des variables qui peuvent être jugées trop sommaires comme le taux d'absentéisme pour le climat social. D'autres données sur la fréquence de cet absentéisme seraient peut être davantage appropriés.

\subsection{Le modèle théorique et les hypothèses de recherche}

\subsubsection{Le modèle théorique testé}

Les construits retenus ayant été précisés, un modèle théorique a été élaboré (Figure 1). Les variables sociales sont considérées comme des variables exogènes et relèvent de cinq domaines distincts : structure/emploi ; rémunérations ; formation; conditions de travail et climat social. La performance économique de l'entreprise fait office de variable endogène.

\section{Figure 1 Modèle testé}

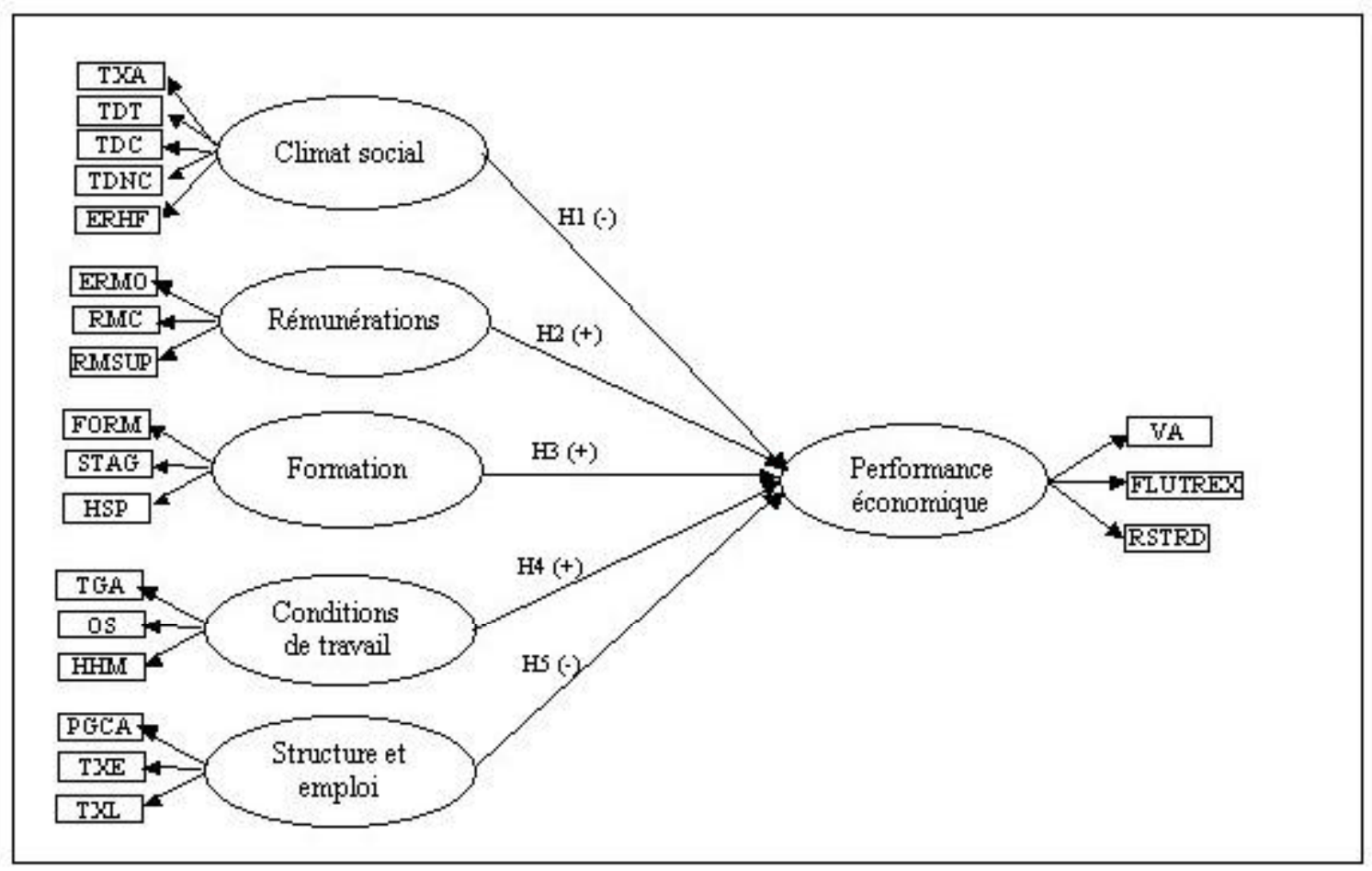

\subsubsection{Les hypothèses de recherche}

Nous allons à présent développer nos cinq hypothèses de recherche sur la base du modèle proposé.

Afin de déterminer l'agencement théorique des variables retenues et le sens des relations, nous nous sommes appuyés sur les recherches existantes.

Les études fondées sur la politique salariale présentées dans notre première partie développent l'importance accordée à la forme de rémunération et son impact sur la performance de l'entreprise. Alors que les résultats ne sont pas clairement établis dans la littérature (Magnan et al, 1993 ; Wilkinson et al, 1994), l'approche la plus fréquente est de considérer que les politiques de rémunérations incitatives contribuent à réduire les coûts d'agence entre les 
salariés et les dirigeants d'une part et les dirigeants et les actionnaires d'autre part et, de ce fait, augmentent la performance de l'entreprise. L'hypothèse testée est donc la suivante :

Hypothèse 1: Plus la politique de rémunération est favorable aux salariés, plus la performance économique de l'entreprise est élevée.

Nous définissons le concept de politique de rémunération favorable aux salariés de la manière suivante :

- niveau de rémunération moyenne des cadres élevé ;

- niveau de rémunération des cadres supérieurs élevés ;

- écart de rémunération cadres / ouvriers faible.

Cette mesure ne rend pas compte, malheureusement, de la constitution de la rémunération (part fixe et part variable et la composition de cette part variable), ce qui en est une limite importante.

Les recherches réalisées selon l'approche des resources based et celles menées sur la politique sociale de l'entreprise (Huselid, 1995 et Grinyer et al, 1990) suggèrent que les pratiques de gestion des ressources humaines en terme de formation peuvent avoir un impact sur la motivation et peuvent conduire à une amélioration des résultats de l'entreprise. En outre, l'ensemble des conditions permettant une amélioration des conditions de travail et un meilleur climat social augmenteraient la productivité et la performance de l'entreprise. Il en découle nos trois hypothèses suivantes :

Hypothèse 2 : Plus la politique de formation est favorable aux salariés, plus la performance économique de l'entreprise est élevée.

Plus une politique de formation est favorable plus :

- les dépenses de formation en pourcentage du chiffre d'affaires sont élevées ;

- le nombre de stagiaires cadres dans l'entreprise est élevé ;

- les heures de stages de formation payées par personne sont élevées.

Ces mesures rendent compte de certaines dimensions de la politique de formation de l'entreprise. Il est vrai cependant que d'autres aspects sont ignorés : la politique de formation est-elle destinée à l'ensemble des salariés ou à quelques uns ? Existe-t-il des suivis de ces formations?

Hypothèse 3 : Plus les conditions de travail sont favorables aux salariés, plus la performance économique de l'entreprise est élevée.

Les conditions de travail sont définies à travers trois variables : le taux de gravité des accidents du travail, le niveau des œuvres sociales de l'entreprise et la durée du travail.

Ainsi, nous considérons que des conditions de travail favorable sont définies par:

- un taux de gravité des accidents du travail faible ;

- des dépenses d'œuvres sociales par rapport à l'effectif élevées ;

- une durée du travail faible.

Hypothèse 4: Plus le climat social est défavorable aux salariés, moins la performance économique de l'entreprise est élevée. 
Le climat social est certainement la dimension la plus délicate à mesurer et nous ne prétendons pas la cerner dans sa totalité mais en apprécier certains aspects. Nous proposons de l'appréhender par cinq variables. A notre sens, un climat social défavorable se traduit par :

- un taux d'absentéisme élevée ;

- un taux de démission total élevé ;

- un taux de démission des cadres élevé ;

- un taux de démission des non cadres élevé ;

- des écarts de rémunérations homme/femme élevés.

Nous faisons l'hypothèse sous-jacente que des niveaux d'absentéisme et de démission donnent une information sur le climat social de l'entreprise, de même que pour l'écart de rémunération hommes/femmes qui indique une forme de discrimination au sein de l'organisation.

Enfin, notre dernière hypothèse est davantage une hypothèse de contrôle sur les différences potentielles entre les secteurs d'activité en fonction de la structure des emplois de l'entreprise et la croissance de l'entreprise en terme d'embauches. Nous pouvons raisonnablement supposer qu'il existe une relation entre la structure de l'entreprise en termes d'emplois et sa performance. La cinquième hypothèse est donc :

Hypothèse 5 : Il existe une relation entre la structure de l'entreprise en terme d'emplois et la performance économique.

La dimension des structures et emplois constitue une dimension de contrôle. Elle est mesurée par trois variables: le pourcentage de cadres dans l'entreprise, les taux d'embauches et de licenciements. Bien que d'autres données serait nécessaires pour appréhender une activité, ces variables renseignent sur le secteur de l'entreprise. Ces secteurs se définissent en partie par leur taux d'embauche, leur taux de licenciement et le pourcentage de salariés cadres par rapport à l'effectif total.

Nous supposons donc que si le pourcentage de cadres est élevé, le taux d'embauches élevé et le taux de licenciement faible, que l'entreprise est en phase de croissance et que, par conséquent, sa performance économique est élevée. En revanche, si le pourcentage de cadres est faible, le taux d'embauche faible et le taux de licenciement élevé, la performance économique sera faible.

\section{Méthodologie de l'étude}

Ce paragraphe décrit l'ensemble de la méthodologie et des choix réalisés afin de collecter et de traiter les informations destinées à tester les hypothèses formulées. Deux contraintes se sont exercées à ce niveau :

- la difficulté d'accès aux données et plus particulièrement aux bilans sociaux ;

- la taille réduite de notre échantillon.

\subsection{Les caractéristiques de l'échantillon}

Notre échantillon présente les caractéristiques suivantes (voir tableau 3) : 
Tableau 3 : Les caractéristiques de notre échantillon

\begin{tabular}{|l|c|c|c|c|c|c|}
\hline Nombre de salariés & 300 à 699 & 700 à 1499 & 1500 à 1999 & 2000 à 4999 & 5000 et + & total \\
\hline$\%$ entreprises & $10.4 \%$ & $25.3 \%$ & $35.6 \%$ & $16.2 \%$ & $12.5 \%$ & $100 \%$ \\
\hline Chiffre d'affaires & 1000 à $2999 \mathrm{k€}$ & 3000 à $5999 \mathrm{k€}$ & 6000 à $9999 \mathrm{k€}$ & $\begin{array}{c}10000 \text { à } \\
14999 \mathrm{k} €\end{array}$ & $\begin{array}{c}15000 \mathrm{k} € \\
\text { et + }\end{array}$ & total \\
\hline$\%$ entreprises & $15.6 \%$ & $25.6 \%$ & $35.4 \%$ & $13.4 \%$ & $10 \%$ & $100 \%$ \\
\hline Secteur d'activité & IAA & ENE & IBI & IBEC & ASE & total \\
\hline$\%$ entreprises & $8 \%$ & $12 \%$ & $30 \%$ & $30 \%$ & $10 \%$ & $100 \%$ \\
\hline
\end{tabular}

IAA = industrie agro-alimentaire; ENE = énergie; IBI = biens intermédiaires; IBEC = biens d'équipement et de consommation; ASE = services extérieurs.

\subsection{Estimation du modèle par la procédure PLS}

Notre modèle théorique a été testé par le biais de l'approche des moindres carrés partiels, développée successivement par Wold (1982) et par Lohmöller (1989). Celle-ci étant à ce jour moins répandue que l'analyse des structures de covariance de type LISREL, il est important d'en présenter l'intérêt et de justifier son utilisation.

Deux raisons principales justifient le recours à l'approche PLS pour notre recherche. Dans l'analyse des structures de covariances, les variables latentes sont indéterminées; leurs variables observées ne reflètent pas totalement leurs structures sous-jacentes. Il en résulte une perte de précision dans la qualité de la prédiction (Valette-Florence, 1993). Par contraste, avec l'approche PLS, la variable latente est une combinaison linéaire exacte des variables observées. La seconde raison est plus pragmatique. Elle tient à la taille de notre échantillon. En effet, l'analyse des structures de covariances implique des hypothèses restrictives en terme de taille de l'échantillon. Ainsi, quand plusieurs centaines d'observations ne sont pas disponibles, les paramètres sont instables. Le risque est alors réel d'aboutir à une absence de résultats (non convergence du modèle) ou à des solutions erronées (variance négative, corrélations supérieures à 1). Par contraste, avec PLS, il n'est pas nécessaire de disposer d'un très grand nombre d'observations. Compte tenu de la taille limitée de notre échantillon $(\mathrm{n}=$ 58), l'utilisation de PLS apparaissait donc comme plus réaliste. Aujourd'hui, de nombreux chercheurs en marketing et en management stratégique privilégient une approche PLS pour des raisons similaires (voir Dawes et Lee, 1996 ; Fornell, 1992 ; Guiot, 1999 ; Tenenhaus, 1998 ; Valette-Florence, 1989).

\section{Les résultats}

\subsection{Purification et qualité des mesures}

En accord avec les procédures recommandées dans la littérature (Churchill, 1979), plusieurs étapes ont été suivies pour vérifier la fiabilité et la validité de nos échelles de mesure.

Dans une première phase, des analyses de fiabilité ont été réalisées. Ce premier examen nous a conduit à supprimer deux concepts (conditions de travail et structure et emploi) car les 
coefficients alpha de Cronbach n'étaient pas acceptables (respectivement $\alpha=0,01$ et $\alpha=0,11$ ) (Nunally, 1978).

Dans une seconde étape, des analyses factorielles en composantes principales avec rotations orthogonales et/ou obliques ont été réalisées sur les variables contenues au sein de chaque hypothèse. Après suppression des items TXA et ERHF, les résultats indiquaient des validités convergente et discriminante satisfaisantes.

En résumé, a l'issue de cette phase de purification notre modèle a été simplifié puisque deux construits ont été supprimés (conditions de travail et structure et emploi). Dès lors, les hypothèses $\mathrm{H} 2$ et $\mathrm{H} 4$ ne seront pas testées dans la suite de cette recherche.

\subsection{Le modèle PLS}

Comme tout modèle d'équations structurelles, les résultats obtenus par le biais d'une approche PLS s'interprètent en deux étapes: (1) évaluation du modèle de mesure, et (2) évaluation du modèle structurel (Fornell et Larcker, 1981).

\subsubsection{Le modèle de mesure}

L'estimation du modèle de mesure correspond à l'évaluation des relations externes entre les variables observables et les variables latentes ${ }^{6}$. Elle dépend de deux principaux critères : la fiabilité et la validité.

Concernant la fiabilité, une première analyse a été effectuée sur chaque item par l'examen des saturations. Puis la fiabilité générale des construits a été évaluée à l'aide du calcul du Rho de Jöreskog ${ }^{7}$. Les indicateurs sont reportés dans le tableau ci-dessous.

Tableau 4 : Propriétés du modèle de mesure PLS

\begin{tabular}{|l|c|c|c|c|}
\hline \multicolumn{1}{|c|}{ Construit } & Indicateur & Loadings & Rho de Jöreskog & $\begin{array}{c}\text { Average Variance } \\
\text { Extracted }\end{array}$ \\
\hline Climat social & TDT & 0,89 & 0,87 & 0,31 \\
& TDC & 0,77 & & \\
& TDNC & 0,82 & 0,74 & 0,50 \\
\hline Rémunérations & ERMO & 0,55 & & \\
& RMC & 0,60 & 0,59 \\
& RMSUP & 0,92 & 0,56 & \\
\hline Formation & FORM & 0,54 & & 0,26 \\
& STAG & 0,95 & 0,90 & \\
\hline Performance & HSP & 0,19 & & \\
économique & VA & 0,86 & & \\
& FLUTREX & 0,83 & & \\
\hline
\end{tabular}

\footnotetext{
${ }^{6}$ Dans le cadre de notre modèle ces relations sont dites réflexives : elles vont des construits aux indicateurs (Fornell et Bookstein, 1982).

${ }^{7}$ Cet estimateur de la fiabilité est considéré comme meilleur que de le coefficient alpha de Cronbach. Contrairement à ce dernier, son calcul ne repose pas sur l'hypothèse d'un poids équivalent des indicateurs dans le construit (Fornell et Larcker, 1981).
} 
L'examen des saturations montre que la fiabilité des énoncés est satisfaisante avec des corrélations supérieures au seuil minimum recommandé de 0,50 (Falk et Miller,1992, p. 81), à l'exception de l'item HSP. Celui-ci n'a pas été supprimé car il aide à définir le construit sousjacent. Les coefficients rho de Jöreskog sont tous supérieurs à 0,55. Ils indiquent une bonne fiabilité générale pour chacun des construits.

Le test de validité d'un construit repose sur les critères de validité convergente et discriminante. Ils peuvent être vérifiés à l'aide de la méthode de Fornell et Larcker (1981).

Deux variables latentes (rémunérations et formation) présentent une validité convergente satisfaisante. Les indices AVE (Average Variance Extracted) sont tous supérieurs à 0,50 (tableau 4). Les construits partagent plus de variance avec leurs indicateurs respectifs qu'avec leurs erreurs de mesure. En revanche, on notera une faible validité convergente pour les concepts de climat social et de performance économique. Cette recherche étant exploratoire, nous avons néanmoins décidé de conserver ces construits.

Les indices root $A V E$ sont reportés en italique sur la diagonale de la matrice des corrélations des variables latentes (tableau 5). Tous sont supérieurs aux valeurs situées en dessous de la diagonale. Les relations entre les variables latentes sont moins fortes que celles entre les construits et leurs variables manifestes.

Tableau 5 : Validité discriminante des concepts

\begin{tabular}{|c|c|c|c|c|}
\hline & Climat social & Rémunérations & Formation & Performance \\
\hline Climat social & $\mathbf{0 , 5 6}$ & & & \\
\hline Rémunérations & 0,05 & $\mathbf{0 , 7 1}$ & & \\
\hline Formation & $-0,01$ & $-0,14$ & $\mathbf{0 , 7 7}$ & \\
\hline Performance & $-0,12$ & $-0,52$ & 0,33 & $\mathbf{0 , 5 1}$ \\
\hline
\end{tabular}

(Note : Les nombres en gras sur la diagonale correspondent à la racine carrée de l'Average Variance Extracted; les nombres sous la diagonale représentent les corrélations entre les construits).

\subsubsection{Le modèle causal}

Dans ce modèle, les liens entre les variables latentes sont estimés afin de tester les hypothèses entre les construits. Le modèle structurel est déterminé à partir des paramètres des relations causales (path coefficients). Ces derniers s'interprètent comme des coefficients de régression. Leur niveau de significativité est vérifié par une procédure de bootstrap (Chin, 1998). Les résultats obtenus sont reportés dans le tableau 6. Ils comprennent les valeurs des paramètres $\mathrm{B}^{8}$ et leurs écarts-type ainsi que les tests de Student.

Tableau 6 : Résultats du test des hypothèses (modèle causal PLS)

\begin{tabular}{|l|c|c|c|c|}
\hline \multicolumn{1}{|c|}{ Hypothèses retenues } & B1* & B2** & Ecart-type & Valeur de t \\
\hline H1 : Climat social $\rightarrow$ performance & $-0,09$ & $-0,12$ & 0,09 & $-1,06$ \\
\hline H2 : Rémunérations $\rightarrow$ performance & 0,47 & 0,51 & 0,22 & $2,09^{*}$ \\
\hline H3 : Formation $\rightarrow$ performance & 0,26 & 0,25 & 0,14 & $1,96^{*}$ \\
\hline
\end{tabular}

* Seuil de signification inférieur à $5 \%$.

\footnotetext{
${ }^{8}$ La lettre B désigne un coefficient standardisé dans le logiciel PLS 1.8
} 
Les résultats des simulations bootstrap montrent la convergence des estimations des paramètres standardisés. Ils attestent de la stabilité des relations observées dans le modèle. L'examen des tests $t$ montre que 2 hypothèses sur 3 sont significatives au seuil de 0,05 .

Nous pouvons également mettre les résultats en perspective au regard du pourcentage de variance expliquée $\left(\mathrm{R}^{2}\right)$ de la variable endogène. Ainsi, le modèle théorique proposé explique $34,4 \%$ de la performance économique.

\subsection{Discussion}

Notre modèle initial comportait 5 hypothèses et 17 variables. Après une première analyse, nous avons dû réduire ce modèle à trois dimensions : les impacts du climat social, de la politique de rémunération et la politique de formation sur la performance de l'entreprise. Ces trois hypothèses ont été testées sur une année, en coupe instantanée.

L'hypothèse sur le climat social n'est pas vérifiée. Le climat social était mesuré par trois variables : le taux de démission total, le taux de démission des cadres et celui des non cadres. Cette absence de corrélation peut s'expliquer par le choix de nos variables, qui masque certains effets. En effet, les démissions peuvent entraîner des gains salariaux immédiats tout en supprimant un certain nombre de coûts cachés. De ce fait, les impacts à court terme ne sont pas clairement établis. La difficulté à mesurer le climat social est donc ici mise en exergue et révèle l'insuffisance de notre mesure.

L'hypothèse sur la politique de rémunération et la performance économique est, en revanche, vérifiée. La politique de rémunération était appréhendée par trois variables : la rémunération moyenne des cadres, les 10 salaires les plus élevés et l'écart moyen entre les salaires cadres et les employés. Ce résultat conforte ceux des études antérieures. En effet, il suggère que la politique salariale, notamment celle menée après des cadres et cadres dirigeants, est associée à une forte productivité. Cette productivité apparaît à travers une forte valeur ajoutée donc dans la performance économique. Soulignons l'intérêt de ce résultat car peu de recherches ont traité de l'impact sur la performance de la politique de rémunération des cadres dirigeants, variable qui est intégrée dans notre construit «rémunération». Cela signifie qu'en France, il existe une forme d'incitation à travers le salaire : ce résultat très répandu aux Etats Unis est plus novateur dans un pays continental, avec la limite qu'il n'intègre pas les rémunérations sous forme de stock options.

Enfin, la dernière hypothèse relative à l'impact de la formation sur la performance est également vérifiée. La politique de formation était mesurée par trois variables : les dépenses de formation, le pourcentage de stagiaires cadres et les heures de stage rémunérées. Cela indiquerait que les investissements entrepris dans ce domaine permettent d'augmenter la performance économique, même à court terme, ce qui n'est pas un résultat évident car l'on pourrait s'attendre à une association positive décalée dans le temps.

\section{Conclusion}

Les travaux antérieurs, à la fois dans la presse académique ou économique, soutiennent que les pratiques de gestion des ressources humaines influencent la performance économique de l'entreprise mais proposent peu de modèles testés empiriquement. Cette étude vient donc modestement compléter la littérature. En effet, dans un premier temps, nous proposons un 
modèle d'hypothèses liant la performance sociale et la performance économique. La performance sociale est appréhendée selon cinq dimensions : le climat social, la politique de rémunération, les conditions de travail, la politique de formation et la structure de l'emploi de l'entreprise. La performance économique est mesurée par trois variables: les flux de trésorerie d'exploitation, la valeur ajoutée et le résultat résiduel. Dans un second temps, ce modèle a été testé sur un échantillon de 58 entreprises françaises, en coupe instantanée et en utilisant les données produites par le bilan social, document établi par toutes les entreprises de plus de 300 salariés. La méthodologie utilisée est PLS, méthode particulièrement adaptée pour des échantillons de taille réduite.

Deux hypothèses sont finalement vérifiées : les relations entre la politique de formation et de rémunération d'une part et la performance économique d'autre part.

L'absence de validité des autres hypothèses met en exergue les limites de notre modèle. En effet, force est de constater qu'il reste délicat d'appréhender le climat social ou les conditions de travail par des indicateurs, même qualitatifs. Par ailleurs, ce modèle n'a été testé que sur une seule année : une approche longitudinale aurait peut être conduit à d'autres résultats et à une remise en questions de la réversibilité possible de la relation testée. Une comparaison internationale pourrait également apporté d'autres pistes car les facteurs historiques et nationaux peuvent contribuer à expliquer les pratiques sociales et salariales des entreprises d'un pays.

De ce fait, il découle plusieurs voies de recherche, tant à partir de ce travail, que d'autres approches en matière sociale et comptable pouvant contribuer à la connaissance de l'impact de la performance sociale des entreprises sur leur performance économique.

\section{Bibliographie}

Abbott W. et Monsen J. (1979), «On the measurement of corporate social responsibility: self-reported disclosures as a method of measuring corporate social involvement », Academy of Management Journal, 22, n³, pp. 501-515.

Alexander G.J. et Buchholz R.A. (1978), «Corporate social responsibility and stock market performance », Academy of Management Journal, 21, n³, pp. 479-486.

Bailey T. (1993), "Discretionary effort and the organisation of work: employee participation and work system reform since Hawthorne", Working paper, Columbia University, New York. Barney (1991), «Firm resources and sustained competitive advantage", Journal of Management, p101.

Bens D.A., Nagar V. et M.H.F. Wong (2002), «Real investment implications of employee stock option exercices », Journal of Accounting Research, vol 40, n² , may, pp 359-393.

Botosan C.A. et Plumlee M.A. (2001), «Stock option expense: the sword of Damocles revealed », Accounting Horizons, Vol 15 n4, December, pp 311-327.

Bowman E. H. et Haire M. (1975), «A Strategic Posture toward Corporate Social Responsibility », California Management Review, pp. 49-58.

Capron M. (1995), «Vers un renouveau de la comptabilité des ressources humaines », Revue Française de Gestion, novembre-décembre, pp. 46-54.

Chin W.W. (1998), The partial least squares approach for structural equation modeling, Modern methods for business research, éd. G.A. Marcoulides, Laurence Erlbaum Associates, Londres, UK. 
Churchill G.A. (1979), "A paradigm for developing better measures of marketing constructs", Journal of Marketing Research, 16, 1, 64-73.

Comhaire P. et Dendauw C. (1998), "Du bilan social vers un tableau de bord social", ANDCP Personnel, n²4, Décembre 1997-Janvier Février 1998, pp 17-28.

D’Arcimoles C.H. (1995), Diagnostic financier et gestion des ressources humaines, nécessité et pertinence du bilan social, Economica.

D'Arcimoles C.H. et Fakhfakh F. (1997) «Licenciements et performance de l'entrperise : une analyse longitudinale 1987-1993 », Journées AGRH et AFC, Vingtième anniversaire du bilan social, Toulouse, 5 et 6 juin, actes pp 63- 75 .

Davidson III W.N. et Worrell D.L. (1990), «A comparison and test of the use of accounting and stock market data in relating corporate social responsibility and financial performance », ABER, Vol 21, n³, fall, pp. 7-19.

Dawes P.L. et Lee D.Y. (1996), "Communication intensity in large-scale organizational high technology purchasing decisions", Journal of Business-to-Business Marketing, 3, 3, 3-34.

Denison D. (1984), "Bringing corporate culture to the bottom line", Organization Dynamics, Autumn, pp 4-23.

Falk R. et Miller N.B. (1992), A primer for soft modelling, Akron, The University of Akron Press.

Flamholtz E. (1971), “A model for Human resource valuation: a stochastic process with service rewards", The Accounting Review, April, pp 253-267.

Fornell C. (1992), "A national customer satisfaction barometer, The swedish experience", Journal of Marketing, 56, 1, 6-21.

Fornell C. et Bookstein F.L. (1982), A Comparative Analysis of Two Structural Equation Models: LISREL and PLS Applied to Market Data, papier de recherche, Ann Arbor, Michigan.

Fornell C. et Larcker D.F. (1981), "Evaluating structural equation models with unobservable variables and measurement errors", Journal of Marketing Research, 18, 2, 39-50.

Freedman M. et Jaggi B. (1992), «An investigation of the long-run relationship between pollution performance and economic performance: the case of pulp and paper firms », Critical Perspectives on Accounting, Vol 3 pp 315-336.

Guiot D. (1999), Biais d'âge subjectif et utilisation symbolique des produits, Thèse de doctorat en sciences de gestion, Université Paris-Dauphine, Paris.

Hackston D. et Milne M.J.(1996), «Some determinants of social and environmental disclosures in New Zealand companies », Accounting, Auditing and Accountability Journal, Vol 9, ${ }^{\circ} 1$, pp. 77-108.

Huselid M.A. (1995), "The impact of human resource management practives on turnover, productivity and corporate financial performance", Academy of Management Journal, Vol 38, $\mathrm{n}^{\circ} 3$, pp 635-672.

Iacono G. (1996), «Une lecture renouvelée du bilan socail une nouvelle piste pour l'emploi ? », ANDCP Personnel, n³71, juillet, pp 44-47.

Lewellen et Huntsman (1970), «Managerial Pay and Corporate Performance », American Economic Review, vol 60, september, pp 710-720.

Lohmöller J.B. (1989), Latent variable path modeling with partial least squares, New York, Springer-Verlag. 
Magnan M., St-Onge et Thorne L. (1993), «L'influence de la taille et de la performance organisationnelle sur la rémunération des dirigeants :une comparaison entre le Canada et les Etats Unis », $4^{\text {ème }}$ Congrès de l'AGRH, Jouy en Josas, 18-19 novembre, pp 99-105.

Masson (1971), «Executive motivations, Earnings, ans Consequenquent Equity Performance", Journal of Political Economy, vol 79, November pp 1278-1292.

Mathews (1997), «Twenty-five years of social and environmental accounting research. Is there a silver jubilee to celebrate ? », Accounting, Auditing and Accountability Journal, Vol $10 \mathrm{~N}^{\circ} 4$, pp. 481-531.

McGuire J. , Chiu C. et Elbing A. (1962), «Executives incomes, sales and profits », American Economic Review, vol 52, september, pp 753-761.

McGuire J.B., Sundgren A. et Schneeweis T. (1988), «Corporate social responsibility and firm financial performance », Academy of Management Journal, Vol 31, n, pp. 854-872.

Nunnally J.C. (1978), Psychometric theory, 2, New York, NY, McGraw-Hill.

Roberts R.W. (1992), «Determinants of corporate social responsibility disclosure: an application of stakeholder theory », Accounting, Organizations and Society, Vol 17, n6, pp. 595-612.

Schuster F. (1988), "Reviving Productivity in America", Personnel Adminsitrator, vol 33, July, pp 65-68.

Sturdivant F.D. et Ginter J.L. (1977), «Corporate social responsiveness, management attitudes and economic performance », California Management Review, Vol XIX, n³, pp. 30-39.

Tenenhaus M. (1998), La régression PLS : Théorie et pratique, Paris, Technip.

Valette-Florence P. (1989), "L'interface vendeur-acheteur: Analyse d'un cas dans les services », Revue Française de Gestion, 75, 28-40.

Valette-Florence P. (1993), Dix années de modèles d'équations structurelles: Un état de l'art, papier de recherche, C.E.R.A.G., 11.

Wilkinson A. , Marchington M., Ackers P. et Goodman J. (1994), "ESOP's fables: a tale of a machine tool company", The International Journal of Human Resource Management, February, pp 121-143.

Wold H. (1982), Soft modeling: The basic design and some extensions, Systems under indirect observation: Causality, structure, prediction, 2, éd. K.G. Jöreskog and H. Wold, Amsterdam, North Holland Publishing Co. 